\title{
Acute high-intensity interval rowing increases thrombin generation in healthy men
}

\author{
Matthew J. Sedgwick ${ }^{1,2} \cdot$ Matthew Thompson $^{1} \cdot$ Jack Garnham $^{1} \cdot$ Alice E. Thackray $^{1}$ • \\ Laura A. Barrett ${ }^{1} \cdot$ Matthew Powis $^{3} \cdot$ David J. Stensel $^{1}$
}

Received: 17 November 2015 / Accepted: 21 March 2016 / Published online: 12 April 2016

(C) The Author(s) 2016. This article is published with open access at Springerlink.com

\begin{abstract}
Purpose High-intensity exercise induces several health benefits, but may acutely and transiently increase the risk of cardiovascular events due to thrombotic changes promoting blood coagulation and thrombin formation. This study examined the effects of high-intensity exercise on plasma thrombin generation and triacylglycerol concentrations.

Methods Sixteen healthy men completed two, 2-day conditions separated by 1 week. On day 1 , participants rested (control) or completed four, 3-min high-intensity rowing intervals at an average rating of perceived exertion of 17 (exercise). Venous blood samples were collected pre- and post-intervention to determine plasma thrombin generation. On day 2, participants rested and consumed a glucose load $(0 \mathrm{~h})$ and high-fat meal $(2 \mathrm{~h})$. Fifteen venous blood samples were collected between 0 and $8 \mathrm{~h}$ to measure plasma thrombin generation and triacylglycerol concentrations.

Results On day 1, lag time was shorter and peak thrombin and endogenous thrombin potential were greater in the exercise than control condition (ES $\geq 0.37$, main effect condition $P \leq 0.03$ ), and post-intervention compared with pre-intervention ( $\mathrm{ES} \geq 0.49$, main effect time $P \leq 0.003)$. The magnitude of the post-intervention change
\end{abstract}

Communicated by Fabio Fischetti.

David J. Stensel

d.j.stensel@lboro.ac.uk

1 School of Sport, Exercise and Health Sciences, Loughborough University, Loughborough, Leicestershire LE11 3TU, UK

2 Department of Sport, Health and Nutrition, Leeds Trinity University, Leeds, UK

3 Pathway Diagnostics Ltd, Surrey, UK was greater in the exercise than control condition for all thrombin generation parameters (condition by time interaction $P \leq 0.05$ ). On day 2 , no differences in postprandial thrombin generation parameters were seen between conditions $(P \geq 0.21)$. The total area under the curve for triacylglycerol was lower in the exercise than control condition (ES $=0.34, P=0.02)$.

Conclusion An acute bout of high-intensity interval rowing increased plasma thrombin generation immediately after exercise, but these differences were eliminated 16-24 $\mathrm{h}$ after exercise.

Keywords Blood coagulation - Cardiovascular disease risk $\cdot$ Exercise intensity $\cdot$ Triacylglycerol

$\begin{array}{ll}\text { Abbreviations } \\ \text { aPTT } & \text { Activated partial thromboplastin time } \\ \text { ES } & \text { Effect size } \\ \text { F1 }+2 & \text { Prothrombin activation fragment } 1+2 \\ \text { RPE } & \text { Rating of perceived exertion } \\ \text { TAG } & \text { Triacylglycerol } \\ \text { TAT } & \text { Thrombin-antithrombin complex } \\ \text { TAUC } & \text { Total area under the curve } \\ \dot{V} \mathrm{O}_{2} & \text { Oxygen uptake }\end{array}$

\section{Introduction}

Blood haemostasis represents a complex interaction between platelets, coagulation and fibrinolysis to maintain the integrity of the vascular wall. An imbalance of this complex regulated system promotes an increased risk of clinical complications from atherosclerotic cardiovascular disease (Borissoff et al. 2011), which remains the leading cause of mortality and morbidity in Western society 
(World Health Organisation 2011). The coagulation system represents an important protagonist in the development of atherogenesis and atherothrombosis (Borissoff et al. 2011). Thrombin is a pivotal enzyme in the coagulation cascade catalysing the conversion of soluble fibrinogen to insoluble fibrin to accelerate clot formation (Crawley et al. 2007). Therefore, lifestyle interventions that improve the coagulation profile by targeting thrombin generation may lower the risk of atherosclerosis and promote important metabolic health benefits.

Endurance training studies highlight the potency of regular exercise to improve markers of coagulation status (Gram et al. 2015; Hilberg et al. 2013). Recent studies demonstrate that low-volume, high-intensity interval exercise elicits a myriad of health benefits that are comparable or even superior to those induced by higher volume, continuous aerobic exercise (Currie et al. 2013; Hood et al. 2011; Little et al. 2011; Trombold et al. 2013). However, acute and strenuous physical exertion may transiently increase the risk of thrombotic events and sudden cardiac death during and immediately after exercise (Thompson et al. 2007). Acute exercise provokes a transient hypercoaguable state (Posthuma et al. 2015), evidenced most consistently by global tests demonstrating a shortening of activated partial thromboplastin time (aPTT) (Gunga et al. 2002; Hilberg et al. 2002, 2003b; Menzel and Hilberg 2011). Furthermore, increases in surrogate markers of thrombin generation such as prothrombin activation fragment $1+2(\mathrm{~F} 1+2)$ and thrombin-antithrombin complex (TAT) have been supported (Hilberg et al. 2003a; Weiss et al. 2002), although this effect may be dependent on the exercise intensity (Cadroy et al. 2002; Menzel and Hilberg 2011; Weiss et al. 1998). The development of thrombin generation assays have made it possible to provide a global assessment of plasma thrombin generation over time, allowing the process of thrombin generation and coagulation potential to be captured with greater sensitivity (Hemker et al. 2003). However, we are not aware of studies that have examined the immediate effect of high-intensity exercise on thrombin generation using this novel technique, highlighting an important gap in our understanding which may have significant implications in determining the safety of high-intensity exercise.

The majority of waking hours are postprandial and, although many postprandial changes are transient in nature, the metabolic disturbances after a meal are unlikely to subside before subsequent meal consumption. Although the activation of coagulation parameters such as factor VII coagulant activity have been supported during postprandial lipaemia (Gill et al. 2001; Silva et al. 2003), evidence of increased postprandial thrombin generation has not been reported yet. Nevertheless, coagulation factor VII appears to be positively associated with postprandial triacylglycerol
(TAG) concentrations (Silva et al. 2003), an established independent risk factor for cardiovascular disease (Nordestgaard et al. 2007), highlighting a potential procoagulant tendency in the postprandial period. Several recent studies highlight the potential efficacy of acute intermittent highintensity exercise performed up to $18 \mathrm{~h}$ before a standardised meal to reduce postprandial TAG concentrations (Freese et al. 2011; Gabriel et al. 2012; Trombold et al. 2013). However, the effect of high-intensity exercise on postprandial thrombin generation the day after exercise is not known.

Therefore, the primary aim of this study was to examine acute changes in plasma thrombin generation immediately after a single session of high-intensity interval rowing exercise using a novel thrombin generation assay in healthy, recreationally active men. In addition, the effect of acute high-intensity interval exercise on postprandial thrombin generation and TAG, glucose and insulin concentrations was determined 16-24 $\mathrm{h}$ after exercise.

\section{Methods}

\section{Participants}

Following approval from the University Ethical Advisory Committee, 16 men aged 19.8-30.2 years were recruited and provided their written informed consent to participate in this study. Participants were non-smokers, not taking medication or dietary supplements and had no known history of cardiovascular disease or coagulation disorders.

\section{Anthropometry}

Stature was measured to the nearest $0.01 \mathrm{~m}$ using a fixed stadiometer (Seca 222, Seca Ltd, Hamburg, Germany), and body mass was quantified to the nearest $0.1 \mathrm{~kg}$ using a digital scale (Adam CFW-150, Milton Keynes, UK). Body mass index was calculated as body mass $(\mathrm{kg})$ divided by stature (m) squared. Waist circumference was determined as the narrowest point of the torso between the xiphoid process and the iliac crest. Triceps, biceps, subscapular and suprailiac skinfold thickness was measured to the nearest $0.2 \mathrm{~mm}$ on the right hand side of the body using skinfold callipers (Baty International, West Sussex, UK) to estimate body density (Durnin and Womersley 1974), and body fat percentage was calculated (Siri 1956).

\section{Preliminary exercise measurements}

Following familiarisation with the stationary rowing ergometer (Model D, Concept II, Nottingham, UK), participants completed a 3 min warm-up, followed immediately 
by four, $3 \mathrm{~min}$ high-intensity rowing intervals. Heart rate was monitored continuously via short-range telemetry (Polar FS20, Polar Electro, Kempele, Finland), and the participant's rating of perceived exertion (RPE) was recorded at the end of each interval using Borg's 6-20 scale (Borg 1973). Participants were instructed to exercise at an intensity equivalent to 10 on the RPE scale during the warm-up (between 'very light' and 'fairly light'), and the remaining intervals corresponded to a self-regulated RPE of 17 ('very hard').

\section{Experimental design}

Participants completed two, 2-day conditions (control and exercise) in a within-measures, counterbalanced, crossover design. The conditions were separated by at least 1 week and the study design is presented schematically in Fig. 1. Participants were asked to refrain from strenuous physical activity and not to consume alcohol or caffeine during the $48 \mathrm{~h}$ period before day 2 of each condition. They also completed a weighed dietary record during the same period of the first condition and replicated this diet before the subsequent condition.

\section{Day 1: intervention day}

Participants arrived at the laboratory at 14:15 following a 2-3 h fast and a venous blood sample was taken after 15 min rest for the measurement of plasma thrombin generation. During the exercise session, participants completed a 3 min warm-up at a self-selected RPE of 10, followed immediately by four, $3 \mathrm{~min}$ rowing intervals at a self-selected RPE of 17 interspersed with 3 min passive recovery. Heart rate was monitored continuously and RPE was recorded at the end of each interval as described previously. Expired air samples were collected during the final minute of intervals 2 and 4 into Douglas bags (Cranlea and Company, Birmingham, UK). Oxygen uptake $(\dot{V}$ $\mathrm{O}_{2}$ ) and carbon dioxide production were analysed using a paramagnetic oxygen analyser and an infrared carbon dioxide analyser (Servomex 1440, East Sussex, UK) and the volume of expired air was quantified using a dry gas meter (Harvard Apparatus Ltd, Kent, UK). Immediately after the cessation of exercise a further venous blood sample was collected. During the control condition, participants rested in the laboratory and a venous blood sample was taken at equivalent time points to the exercise condition.

\section{Day 2: postprandial day}

Participants reported to the laboratory at 07:45 following a $10 \mathrm{~h}$ overnight fast and a fasting venous blood sample was taken after 15 min rest to determine plasma thrombin generation and TAG, glucose and insulin concentrations. They then consumed a $75 \mathrm{~g}$ glucose load $(82.5 \mathrm{~g}$ dextrose monohydrate) in $300 \mathrm{ml}$ water for breakfast, marking the start of the postprandial period. This breakfast was selected to represent the carbohydrate-rich breakfasts that are typically consumed in Westernised countries (Reeves et al. 2013), and to investigate the effect of high-intensity interval rowing on markers of insulin resistance and sensitivity. Subsequent venous blood samples were collected at 0.25 , $0.5,0.75,1,1.5,2,2.5,3,3.5,4,5,6,7$ and $8 \mathrm{~h}$ after the ingestion of the glucose load. Plasma thrombin generation parameters were measured from samples collected at $0,2,5$ and $8 \mathrm{~h}$. Concentrations of TAG and glucose were measured from all samples, and insulin was measured from all samples except at 5 and $7 \mathrm{~h}$. Participants consumed a standardised meal within $20 \mathrm{~min}$ immediately after the venous blood sample at $2 \mathrm{~h}$. The meal consisted of white bread, butter, cheese, potato crisps, whole milk and chocolate milkshake powder, and provided $0.7 \mathrm{~g}$ fat $(51 \%$ of total meal energy), $1.2 \mathrm{~g}$ carbohydrate (37 \%), $0.4 \mathrm{~g}$ protein $(12 \%)$ and $55 \mathrm{~kJ}$ energy per kilogram body mass. The time taken to consume the meal during the first condition was replicated in the remaining condition. Participants consumed water ad libitum between 2 and $8 \mathrm{~h}$ during the first
Fig. 1 Diagram of the 2-day study protocol. TAG, triacylglycerol

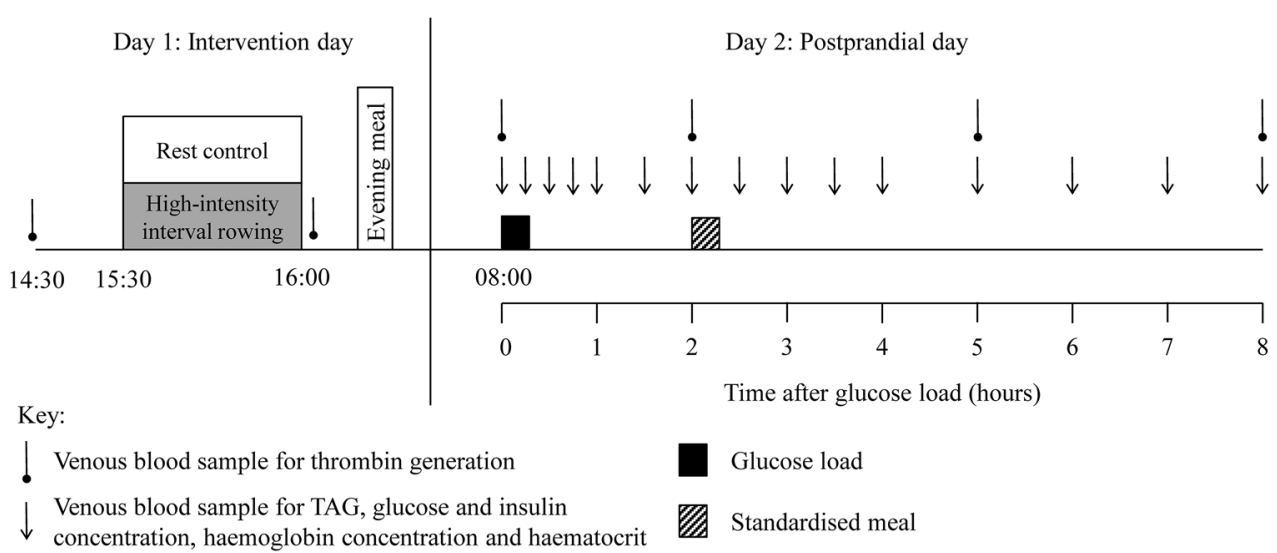


condition; the ingested volume was replicated in the subsequent condition.

\section{Analytical methods}

Venous blood samples were collected via venepuncture from an antecubital vein on day 1 , and on day 2, using a cannula (Venflon, Becton-Dickinson, Helsingborg, Sweden) inserted into an antecubital vein. All samples were collected in the semi-supine position. For samples collected using a cannula, patency was maintained by flushing with non-heparinised saline [0.9\% (w/v) sodium chloride; Baxter Healthcare Ltd, Norfolk, UK]. Residual saline was discarded using a $2 \mathrm{~mL}$ syringe prior to sample collection. Samples were drawn into pre-chilled $5 \mathrm{~mL}$ citrate theophylline adenosine dipyridamole (CTAD) monovettes (Sarstedt, Leicester, UK) for the assessment of plasma thrombin generation on day 1 and day 2 . To prevent the degradation of clotting factors, nine parts venous blood was added to one part of $0.106 \mathrm{M}$ trisodium citrate (equivalent to $3.2 \%$ trisodium citrate). Monovettes were immediately centrifuged at $1500 \times g$ for $10 \mathrm{~min}$ at $4{ }^{\circ} \mathrm{C}$ (Heraeus Labofuge 400R, Thermo Electron, Osterode, Germany). The top $2 \mathrm{~mL}$ of plasma supernatant was evenly aliquoted into two Eppendorf tubes (Eppendorf, Hamburg, Germany) and recentrifuged under the same conditions. Subsequently, the top $500 \mu \mathrm{L}$ of plasma supernatant from each tube was dispensed into Eppendorf tubes to obtain platelet-poor plasma, and samples were stored at $-20{ }^{\circ} \mathrm{C}$ for subsequent analysis. Thrombin generation was measured in duplicate using a commercially available fluorogenic assay kit (Technothrombin ${ }^{\circledR}$ TGA, Technoclone, Vienna, Austria) on a fully automated coagulation analyser (Ceveron ${ }^{\circledR}$ Alpha, Technoclone, Vienna, Austria). The thrombin generation vs. time curve was established and the following parameters of thrombin activity were recorded: (1) lag time: time until thrombin burst; (2) peak thrombin: maximal concentration of thrombin formed; (3) endogenous thrombin potential: total enzymatic work performed by thrombin equated as the area under the thrombin generation curve. The interand intra-assay coefficient of variation was 7.7 and $4.5 \%$, respectively.

To determine the concentration of TAG, glucose and insulin on day 2, venous blood was collected into prechilled $9 \mathrm{~mL}$ EDTA monovettes (Sarsedt, Leicester, UK). Monovettes were centrifuged immediately at $1500 \times \mathrm{g}$ for $10 \mathrm{~min}$ at $4{ }^{\circ} \mathrm{C}$ (Heraeus Labofuge 400R, Thermo Electron, Osterode, Germany). The plasma supernatant was aliquoted into Eppendorf tubes prior to storage at $-20{ }^{\circ} \mathrm{C}$ for subsequent analysis. Plasma TAG and glucose concentration was analysed by enzymatic, colorimetric methods using a benchtop analyser (Pentra 400, HORIBA ABX Diagnostics, Montpellier, France). Plasma insulin concentration was analysed using a commercially available enzymelinked immunoassay (Mercodia Insulin ELISA, Mercodia AB, Uppsala, Sweden). The within-batch coefficient of variation for TAG, glucose and insulin concentration was $1.7 \%\left(1.20 \mathrm{mmol} \mathrm{L}^{-1}\right), 0.5 \%\left(7.19 \mathrm{mmol} \mathrm{L}^{-1}\right)$ and $4.7 \%$ (186.5 pmol L $\left.{ }^{-1}\right)$, respectively.

At each blood sampling point, haemoglobin concentration and haematocrit were quantified in duplicate to estimate acute changes in plasma volume (Dill and Costill 1974). Haemoglobin concentration was assessed using the cyanmethemoglobin method; $20 \mu \mathrm{L}$ whole blood was added to $5 \mathrm{~mL}$ Drabkin's solution and the absorbance was quantified photometrically at a wavelength of $546 \mathrm{~nm}$ (Cecil CE1011, Cecil Instruments, Cambridge, UK). Haematocrit was quantified using a microhaemoatocrit centrifuge and reader (Haematospoin 1300 Microcentrifuge, Hawksley and Sons Ltd, Sussex, UK).

\section{Statistical analysis}

Data were analysed using the IBM SPSS Statistics software for Windows version 21.0 (IBM Corporation, New York, USA). Descriptive statistics illustrating the physical and physiological characteristics and exercise responses were calculated. The trapezium rule was used to calculate time-averaged total area under the curve (TAUC) values. The homeostasis model assessment of insulin resistance (HOMA-IR) (Matthews et al. 1985) and insulin sensitivity index (Matsuda and DeFronzo 1999) were calculated. Normality of the data was checked using Shapiro-Wilk tests. Normally distributed data are presented as mean (SD). Thrombin generation parameters, and TAG, glucose and insulin concentrations were not normally distributed and were natural $\log$ transformed prior to analysis. These data are presented as geometric mean $(95 \%$ confidence interval) and analysis is based on ratios of the geometric means and $95 \%$ confidence intervals for ratios.

Linear mixed models repeated for condition (control and exercise) and time (pre- and post-intervention) were used to examine differences in thrombin generation parameters on day 1. Dietary intake, markers of insulin resistance and sensitivity, fasting concentrations and TAUC responses on day 2 were analysed using linear mixed models with condition (control and exercise) as the sole factor. Differences in thrombin generation parameters and TAG, glucose and insulin concentrations over the total $8 \mathrm{~h}$ postprandial period were examined using linear mixed models repeated for condition (control and exercise) and time. All linear mixed models included a random effect for each participant.

Statistical significance was accepted as $P<0.05$ and absolute standardised effect sizes (ES) are included to supplement important findings. In the absence of a clinical anchor, an ES of 0.2 was considered the minimum 
important difference in all outcome measures, 0.5 moderate and 0.8 large (Cohen 1988). Graphical representations of results are presented as mean (SEM) to avoid distortion of the graphs.

\section{Results}

\section{Participant characteristics}

The physical characteristics of participants were as follows: age 23.4 (2.9) years, body mass 80.9 (13.9) kg, body mass index 24.7 (3.6) $\mathrm{kg} \mathrm{m}^{-2}$, body fat 18.5 (4.2) \%, waist circumference $84.6(10.1) \mathrm{cm}$.

\section{Dietary intake}

Average energy intake was similar during the $48 \mathrm{~h}$ before day 2 of the control and exercise conditions [10.1 (2.9) vs. 10.3 (3.3) $\mathrm{MJ} \mathrm{day}^{-1}$, respectively; main effect condition $P=0.38$ ]. Average 2-day macronutrient intake did not differ between the control and exercise conditions for protein [106 (26) vs. 113 (34) $\mathrm{g} \mathrm{day}^{-1}$; main effect condition $P=0.22]$, carbohydrate [299 (116) vs. 302 (119) $\mathrm{g} \mathrm{day}^{-1}$; main effect condition $P=0.67$ ] or fat [94 (39) vs. 96 (44) $\mathrm{g} \mathrm{day}^{-1}$; main effect condition $\left.P=0.62\right]$, respectively.

\section{Rowing exercise responses}

Participants completed the four high-intensity rowing bouts at a mean power output of 224 (49) W, and covered a mean distance of 772 (56) $\mathrm{m}$ per interval [3087 (224) m in total]. Mean $\dot{V} \mathrm{O}_{2}$ during the exercise session was 3.36 (0.76) $\mathrm{L} \mathrm{min}^{-1}$, corresponding to 42 (9) $\mathrm{mL} \mathrm{kg}^{-1} \mathrm{~min}^{-1}$, and the average respiratory exchange ratio was 1.05 (0.09). Mean heart rate was 179 (10) beats $\mathrm{min}^{-1}$, which represented 91 (5) \% of age predicted maximum heart rate, and the average RPE was 17 (0) ('very hard' on the scale).

\section{Day 1: thrombin generation parameters pre- and post-intervention}

Plasma thrombin generation parameters measured pre- and post-intervention in the control and exercise conditions are displayed in Fig. 2. Lag time was $8 \%$ lower in the exercise compared with the control condition $(-15$ to $-1 \%$, $\mathrm{ES}=0.37$, main effect condition $P=0.03)$, and $11 \%$ lower post-intervention compared with pre-intervention $(-17$ to $-4 \%, \mathrm{ES}=0.49$, main effect time $P=0.003)$. The magnitude of reduction was greater in the exercise than control condition ( -17 vs. $-4 \%$, respectively; condition by time interaction $P=0.05$ ).
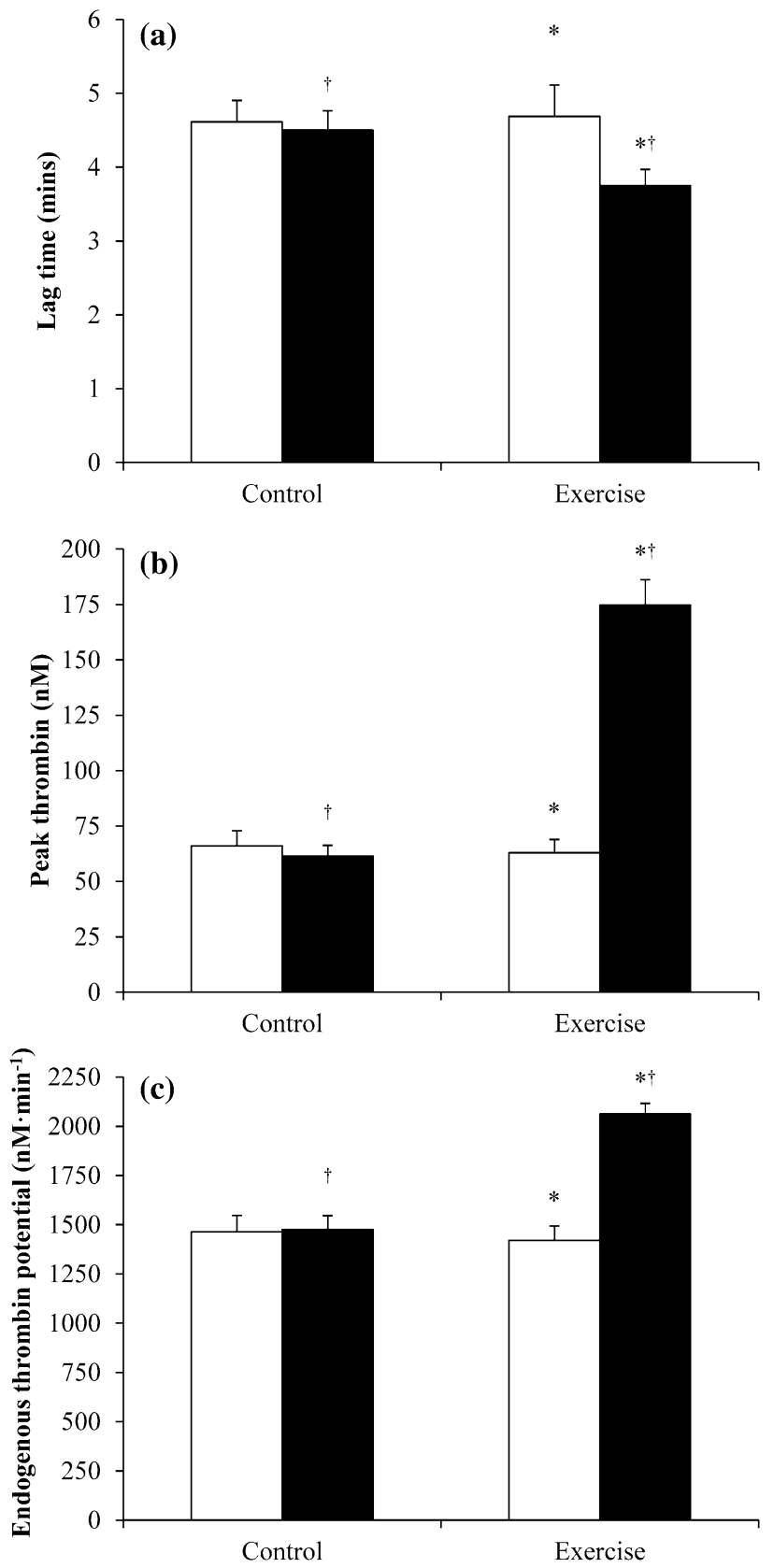

Fig. 2 Plasma thrombin generation parameters measured pre- (open square) and post- (closed square) intervention in the control and exercise conditions $(n=16)$ : a lag time; $\mathbf{b}$ peak thrombin; $\mathbf{c}$ endogenous thrombin potential. Values are mean (SEM). *Significant difference between exercise and control conditions, main effect condition $P \leq 0.03$; ${ }^{\dagger}$ significant difference between pre- and post-intervention, main effect time $P \leq 0.003$

Peak thrombin was $66 \%$ higher in the exercise compared with the control condition (46 to $88 \%, \mathrm{ES}=1.37$, main effect condition $P<0.001$ ), and $67 \%$ higher postintervention compared with pre-intervention (48 to $90 \%$, $\mathrm{ES}=1.39$, main effect time $P<0.001$ ). The magnitude of change was greater in the exercise than control condition 
(184 vs. $-3 \%$, respectively; condition by time interaction $P<0.001)$.

Endogenous thrombin potential was $17 \%$ higher in the exercise compared with the control condition (9 to $25 \%$, $\mathrm{ES}=0.75$, main effect condition $P<0.001$ ), and $23 \%$ higher post-intervention compared with pre-intervention (15 to $32 \%, \mathrm{ES}=0.99$, main effect time $P<0.001)$. The magnitude of increase was greater in the exercise than control condition (47 vs. $2 \%$ respectively; condition by time interaction $P<0.001)$.

\section{Day 2: fasting thrombin generation and plasma concentrations}

Fasting plasma thrombin generation parameters and TAG, glucose and insulin concentrations are displayed in Table 1. Linear mixed models revealed no significant differences in fasting plasma thrombin generation between the control and exercise condition for lag time (main effect condition $P=0.87$ ), peak thrombin (main effect condition $P=0.93$ ) or endogenous thrombin potential (main effect condition $P=0.46$ ). Fasting plasma TAG concentration was $13 \%$ lower in the exercise compared with the control condition $(\mathrm{ES}=0.46$, main effect condition $P=0.01$ ). There was no significant difference in fasting plasma glucose (main effect condition $P=0.93$ ) or insulin (main effect condition $P=0.75$ ) concentration between the control and exercise conditions. No differences were seen between the control and exercise conditions for HOMA-IR [1.29 (0.50) vs. 1.25 (0.47), respectively; $95 \% \mathrm{CI}-0.26$ to 0.18 , main effect condition $P=0.68]$ or the insulin sensitivity index [6.65 (2.20) vs. 6.80 (2.55), respectively; $95 \% \mathrm{CI}-1.07$ to 1.36 , main effect condition $P=0.80]$.

\section{Day 2: postprandial thrombin generation and plasma concentrations}

No significant differences were seen between the control and exercise conditions for any measure of thrombin generation in the postprandial period (main effect condition $P \geq 0.14$; condition by time interaction $P \geq 0.73$ ). Linear mixed models revealed a significant main effect of time for lag time $(P=0.01)$ and endogenous thrombin potential $(P=0.02)$, but not peak thrombin $(P=0.09)$. Specifically, lag time was lower at $8 \mathrm{~h}$ compared with 0 and $2 \mathrm{~h}(\sim 11 \%$; $P \leq 0.01)$, and endogenous thrombin potential was elevated at 2, 5 and $8 \mathrm{~h}$ compared with $0 \mathrm{~h}(\sim 11 \% ; P \leq 0.01)$. No significant difference in TAUC responses was identified between the control and exercise conditions for any measure of thrombin generation (main effect condition: lag time $P=0.79$; peak thrombin $P=0.21$; endogenous thrombin potential $P=0.22$ ) (Table 1).
Plasma TAG, glucose and insulin responses over the postprandial period for the experimental conditions are shown in Fig. 3. Linear mixed models identified differences in postprandial plasma TAG concentrations over time between conditions (main effect condition $P<0.001$; main effect time $P<0.001$; condition by time interaction $P=0.99$ ). Mean TAG concentration was $12 \%$ lower in the exercise compared with the control condition $(-15$ to $-9 \%, \mathrm{ES}=0.29$, main effect condition $P<0.001)$. The TAG TAUC was $11 \%$ lower in the exercise compared with the control condition $(\mathrm{ES}=0.34$, main effect condition $P=0.02$ ) (Table 1).

Linear mixed models revealed no differences in postprandial plasma glucose concentration between the conditions (main effect condition $P=0.28$; main effect time $P<0.001$; condition by time interaction $P=1.00)$. No significant difference was observed in glucose TAUC between the conditions (main effect condition $P=0.38$ ) (Table 1).

Postprandial plasma insulin concentration did not differ significantly between the conditions (main effect condition $P=0.39$; main effect time $P<0.001$; condition by time interaction $P=0.99$ ). The insulin TAUC was similar between the control and exercise conditions (main effect condition $P=0.69$ ) (Table 1).

\section{Discussion}

The primary finding from the present study was that a single session of high-intensity interval rowing increased plasma thrombin generation immediately after exercise in healthy, recreationally active men. However, no differences in postprandial plasma thrombin generation were seen the next day 16-24 h after exercise, but a single bout of interval rowing exercise resulted in a small reduction in postprandial TAG concentrations the next day.

The shortening of lag time immediately after the rowing intervals supports previous studies reporting a shortening of plasma clotting times (e.g., aPTT) after a single session of exercise (Gunga et al. 2002; Hilberg et al. 2002, 2003b; Menzel and Hilberg 2011). However, plasma clot formation occurs before the majority ( $95 \%$ ) of thrombin is generated and, therefore, thrombin generating processes that take place after clotting has occurred are not reflected in the clotting time (Hemker and Béguin 1995). The substantial increase in the concentration of peak thrombin immediately after the high-intensity rowing intervals appears to be a novel finding. Indirect evidence of increased thrombin generation can be provided by studies reporting post-exercise increases in surrogate markers such as $\mathrm{F} 1+2$ and TAT (Hilberg et al. 2003a; Weiss et al. 2002); however, this finding is not universal (Eriksson-Berg et al. 2002), but appears more pronounced at higher exercise intensities (Cadroy 
Table 1 Fasting and timeaveraged total area under the postprandial concentration vs. time curve in the control and exercise conditions on day 2

\begin{tabular}{|c|c|c|c|c|}
\hline & Control & Exercise & $\begin{array}{l}\text { Control vs. exercise } \\
95 \% \mathrm{CI}^{\mathrm{a}}\end{array}$ & Effect size \\
\hline \multicolumn{5}{|l|}{ Time lag } \\
\hline Fasting (min) & $4.29(3.75-4.91)$ & $4.33(3.78-4.96)$ & -11 to 14 & 0.04 \\
\hline TAUC (min) & $4.12(3.68-4.63)$ & $4.18(3.72-4.68)$ & -8 to 12 & 0.06 \\
\hline \multicolumn{5}{|c|}{ Peak thrombin concentration } \\
\hline Fasting (nM) & $73.5(55.4-97.3)$ & $74.4(56.1-98.6)$ & -26 to 39 & 0.03 \\
\hline TAUC (nM) & 89.5 (76.4-104.9) & $80.3(68.5-94.1)$ & -25 to 7 & 0.36 \\
\hline \multicolumn{5}{|c|}{ Endogenous thrombin potential } \\
\hline Fasting $\left(\mathrm{nM} \mathrm{min} \min ^{-1}\right)$ & $1558(1360-1785)$ & $1475(1288-1690)$ & -19 to 11 & 0.23 \\
\hline TAUC $\left(\mathrm{nM} \mathrm{min}{ }^{-1}\right)$ & $1708(1571-1857)$ & $1628(1497-1770)$ & -12 to 3 & 0.32 \\
\hline \multicolumn{5}{|l|}{ Triacylglycerol } \\
\hline Fasting $\left(\mathrm{mmol} \mathrm{L}^{-1}\right)$ & $0.96(0.81-1.13)$ & $0.83(0.71-0.99)$ & -21 to $-4^{\mathrm{b}}$ & 0.46 \\
\hline TAUC $\left(\mathrm{mmol} \mathrm{L}^{-1}\right)$ & $1.28(1.05-1.55)$ & $1.13(0.93-1.37)$ & -20 to $-2^{b}$ & 0.34 \\
\hline \multicolumn{5}{|l|}{ Glucose } \\
\hline Fasting $\left(\mathrm{mmol} \mathrm{L}^{-1}\right)$ & $5.53(5.34-5.72)$ & $5.53(5.35-5.73)$ & -3 to 3 & 0.02 \\
\hline TAUC $\left(\mathrm{mmol} \mathrm{L}^{-1}\right)$ & $5.69(5.41-5.98)$ & $5.81(5.53-6.10)$ & -3 to 7 & 0.19 \\
\hline \multicolumn{5}{|l|}{ Insulin } \\
\hline Fasting (pmol L ${ }^{-1}$ ) & $28.9(23.2-36.1)$ & $28.3(22.6-35.3)$ & -16 to 14 & 0.05 \\
\hline TAUC $\left(\mathrm{pmol} \mathrm{L}^{-1}\right)$ & $156(131-185)$ & $151(127-180)$ & -18 to 14 & 0.11 \\
\hline
\end{tabular}

Values are geometric mean (95\% confidence interval) for $n=16$. Statistical analyses are based on natural $\log$ transformed data. Comparisons were made using linear mixed models

TAUC time-averaged total area under the concentration vs. time curve

a $95 \%$ confidence interval for the ratio of geometric means

b Significant difference between exercise and control conditions $(P<0.05)$ et al. 2002; Menzel and Hilberg 2011; Weiss et al. 1998) such as the exercise is in the present study.

In contrast to the findings of the present study, previous exercise studies did not observe an increase in endogenous thrombin potential immediately after exercise (Hilberg et al. 2002, 2003a, b). This discrepancy may reflect differences in exercise intensity and duration, but it is more likely due to methodological differences in the measurement of thrombin generation with previous studies using a chromogenic, rather than a fluorigenic, substrate and adjusting for $\alpha_{2}$-macroglobulin-bound thrombin (Hilberg et al. 2002, 2003a, b). Nevertheless, endogenous thrombin potential represents the most robust measure of thrombin generation (Al Dieri et al. 2012), and the findings of the present study suggest that a single session of high-intensity interval rowing provokes a transient prothrombotic tendency.

The present study provides a potential mechanism for the transient increase in risk of cardiovascular complications during and immediately after acute high-intensity exercise. This adds to the debate concerning the potential safety of high-intensity exercise. Although it is wellestablished that high-intensity exercise induces a transient hypercoaguable state (Posthuma et al. 2015), the risk of acute exercise-induced cardiovascular events is low (Albert et al. 2000; Rognmo et al. 2012). Furthermore, regular exercise training improves markers of coagulation status (Gram et al. 2015; Hilberg et al. 2013), and there is no evidence that the reported health benefits of high-intensity exercise (Currie et al. 2013; Hood et al. 2011; Little et al. 2011; Trombold et al. 2013) are outweighed by the risks (Thompson et al. 2007).

The high-intensity rowing session demonstrated efficacy in reducing postprandial TAG concentrations 16-24 h post-exercise, but elicited no significant or meaningful change in postprandial thrombin generation. While the exact time course of elevated blood coagulation potential in the hours after acute exercise is not known, deleterious perturbations in aPTT, F1 +2 and TAT appear to subside 3-6 $\mathrm{h}$ after strenuous exercise lasting $1 \mathrm{~h}$ in duration (Weiss et al. 2002). Previous studies have demonstrated that meal consumption stimulates a transient hypercoaguable state, primarily supported by the activation of factor VII (Gill et al. 2001; Silva et al. 2003), which appears to be positively associated with postprandial TAG concentrations (Silva et al. 2003). In the present study, the small increase in endogenous thrombin potential during the postprandial period in both conditions suggests meal consumption may translate to increased thrombin generation. However, postprandial endogenous thrombin potential was not different between the conditions, suggesting that the 


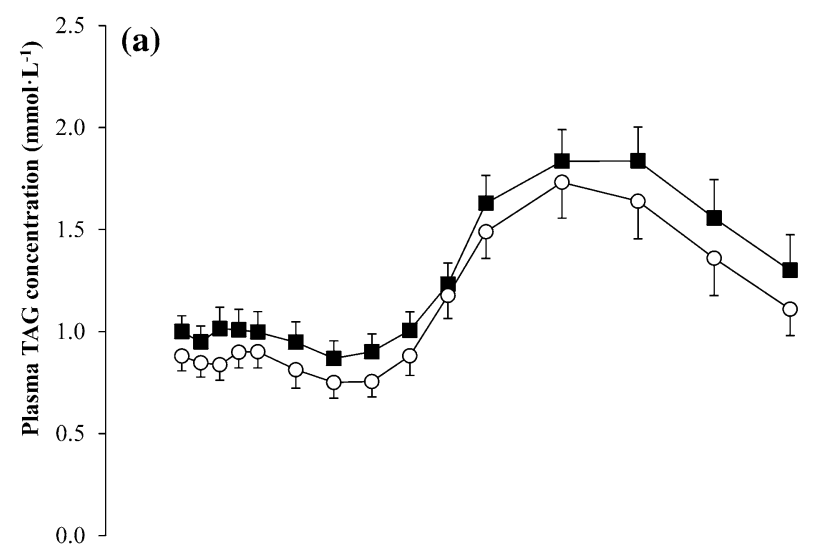

et al. 2013), although others have demonstrated no change in the postprandial lipaemic response to high-intensity exercise (Allen et al. 2014; Tan et al. 2013). The potential for high-intensity interval rowing to reduce postprandial lipaemia is encouraging considering elevated postprandial TAG concentrations are a strong independent risk factor for cardiovascular disease (Nordestgaard et al. 2007). Furthermore, lack of time and enjoyment are commonly cited barriers to exercise participation (Trost et al. 2002); therefore, high-intensity exercise may offer a time-efficient and for some a more enjoyable strategy to induce metabolic health benefits.

A limitation of the present study is that no markers of
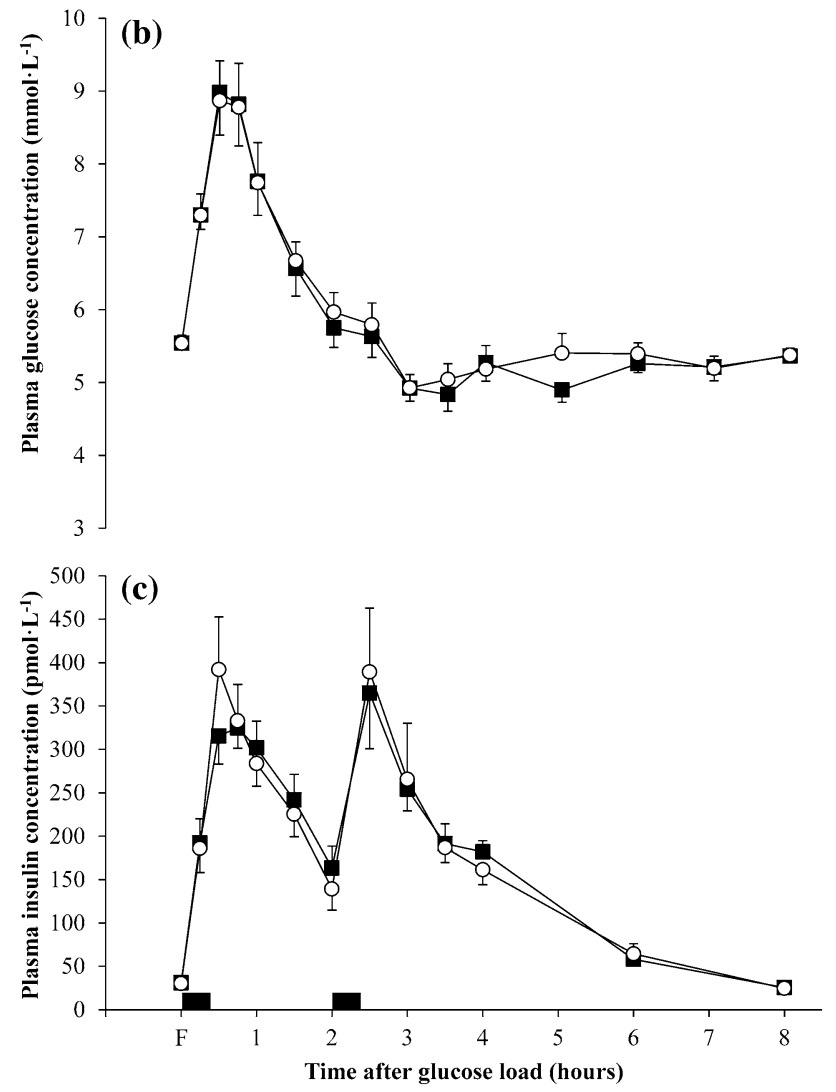
fibrinolysis were measured. Several studies have reported that the transient increase in blood coagulation after exercise is matched or even exceeded by augmented fibrinolysis (Hilberg et al. 2003b; Weiss et al. 1998). A further limitation concerns the recruitment of healthy, recreationally active men. Additional research should be conducted to examine if similar responses are observed in patient populations or those who are unaccustomed to high-intensity exercise. However, despite these limitations, this study has provided a meaningful and important insight into the effects of acute high-intensity interval rowing on thrombin generation using a novel assay which is not available currently in the literature.

In conclusion, acute high-intensity interval rowing provokes a transient elevation in plasma thrombin generation immediately after exercise in healthy, recreationally active men. However, postprandial thrombin generation parameters were not different the next day, 16-24 $\mathrm{h}$ after exercise, despite a reduction in postprandial plasma TAG concentrations.

Acknowledgments The authors thank all of the volunteers for their participation in this study. The research was supported by the National Institute for Health Research (NIHR) Diet, Lifestyle and Physical Activity Biomedical Research Unit based at University Hospitals of Leicester and Loughborough University. The views expressed are those of the authors and not necessarily those of the NHS, the NIHR or the Department of Health.

Fig. 3 Fasting (F) and postprandial plasma concentrations in the control (closed square) and exercise (open circle) conditions $(n=16)$ : a triacylglycerol (TAG); b glucose; $\mathbf{c}$ insulin. Values are mean (SEM). Black rectangles denote consumption of a glucose load and standardised meal at 08:00 and 10:00, respectively. There was a significant main effect of condition for TAG $(P<0.001)$

exercise-induced changes in postprandial TAG concentrations are independent of changes in endogenous thrombin potential.

Several recent studies have reported reductions in postprandial TAG concentrations after intermittent high-intensity running and cycling (Ferreira et al. 2011; Freese et al. 2011; Gabriel et al. 2012, 2013; Tan et al. 2014; Trombold

\section{Compliance with ethical standards}

Ethical standards All procedures performed in studies involving human participants were in accordance with the ethical standards of the institutional and national research committee and with the 1964 Helsinki declaration and its later amendments.

Conflict of interest The authors declare that they have no conflict of interest.

Open Access This article is distributed under the terms of the Creative Commons Attribution 4.0 International License (http://creativecommons.org/licenses/by/4.0/), which permits unrestricted use, distribution, and reproduction in any medium, provided you give appropriate credit to the original author(s) and the source, provide a link to the Creative Commons license, and indicate if changes were made. 


\section{References}

Al Dieri R, de Laat B, Hemker HC (2012) Thrombin generation: what have we learned? Blood Rev 26:197-203

Albert CM, Mittleman MA, Chae CU, Lee IM, Hennekens CH, Manson JE (2000) Triggering of sudden death from cardiac causes by vigorous exertion. N Engl J Med 343:1355-1361

Allen E, Gray P, Kollias-Pearson A, Oag E, Pratt K, Henderson J, Gray SR (2014) The effect of short-duration sprint interval exercise on plasma postprandial triacylglycerol levels in young men. J Sports Sci 32:911-916

Borg GAV (1973) Perceived exertion: a note on "history" and methods. Med Sci Sports 5:90-93

Borissoff JI, Spronk HMH, ten Carte H (2011) The hemostatic system as a modulator of atherosclerosis. N Engl J Med 364:1746-1760

Cadroy Y, Pillard F, Sakariassen KS, Thalamas C, Boneu B, Riviere D (2002) Strenuous but not moderate exercise increases the thrombotic tendency in healthy sedentary male volunteers. J Appl Physiol 93:829-833

Cohen J (1988) Statistical power analysis for the behavioural sciences, 2nd edn. Lawrence Erlbaum Associates, Hillsdale

Crawley JTB, Zanardelli S, Chion CKNK, Lane DA (2007) The central role of thrombin in hemostasis. J Thromb Haemost 5(Suppl 1):95-101

Currie KD, Dubberley JB, McKelvie RS, MacDonald MJ (2013) Low-volume, high-intensity interval training in patients with CAD. Med Sci Sports Exerc 45:1436-1442

Dill DB, Costill DL (1974) Calculation of percentage changes in volumes of blood, plasma, and red cells in dehydration. J Appl Physiol 37:247-248

Durnin JV, Womersley J (1974) Body fat assessed from total body density and its estimation from skinfold thickness: measurements on 481 men and women aged from 16 to 72 years. Br J Nutr 32:77-97

Eriksson-Berg M, Egberg N, Eksborg S, Schenck-Gustafsson K (2002) Retained fibrinolytic response and no coagulation activation after acute physical exercise in middle-aged women with previous myocardial infarction. Thromb Res 105:481-486

Ferreira AP, Ferreira CB, Souza VC, Córdova CO, Silva GC, Nóbrega Ode T, França NM (2011) The influence of intense intermittent versus moderate continuous exercise on postprandial lipemia. Clinics (Sao Paulo) 66:535-541

Freese EC, Levine AS, Chapman DP, Hausman DB, Cureton KJ (2011) Effects of acute sprint interval cycling and energy replacement on postprandial lipemia. J Appl Physiol 111:1584-1589

Gabriel B, Ratkevicius A, Gray P, Frenneaux MP, Gray SR (2012) High-intensity exercise attenuates postprandial lipaemia and markers of oxidative stress. Clin Sci (Lond) 123:313-321

Gabriel BM, Pugh J, Pruneta-Deloche V, Moulin P, Ratkevicius A, Gray SR (2013) The effect of high intensity interval exercise on postprandial triacylglycerol and leukocyte activation-monitored for $48 \mathrm{~h}$ post exercise. PLoS One 8:e82669

Gill JMR, Frayn KN, Wootton SA, Miller GJ, Hardman AE (2001) Effects of prior moderate exercise on exogenous and endogenous lipid metabolism and plasma factor VII activity. Clin Sci (Lond) 100:517-527

Gram AS, Bladbjerg EM, Skov J, Ploug T, Sjödin A, Rosenkilde M, Madsen DE, Stallknecht BM (2015) Three months of strictly controlled daily endurance exercise reduces thrombin generation and fibrinolytic risk markers in younger moderately overweight men. Eur J Appl Physiol 115:1331-1338

Gunga HC, Kirsch K, Beneke R, Böning D, Hopfenmüller W, Leithäuser R, Hütler M, Röcker L (2002) Markers of coagulation, fibrinolysis and angiogenesis after strenuous short-term exercise (Wingate-test) in male subjects of varying fitness levels. Int J Sports Med 23:495-499

Hemker HC, Béguin S (1995) Thrombin generation in plasma: its assessment via the endogenous thrombin potential. Thromb Haemost 74:134-138

Hemker HC, Giesen P, Al Dieri R, Regnault V, de Smedt E, Wagenvoord R, Lecompte T, Béguin S (2003) Calibrated automated thrombin generation measurement in clotting plasma. Pathophysiol Haemost Thromb 33:4-15

Hilberg T, Prasa D, Stürzebecher J, Gläser D, Gabriel HHW (2002) Thrombin potential and thrombin generation after exhaustive exercise. Int J Sports Med 23:500-504

Hilberg T, Gläser D, Reckhart C, Prasa D, Stürzebecher J, Gabriel HHW (2003a) Blood coagulation and fibrinolysis after longduration treadmill exercise controlled by individual anaerobic threshold. Eur J Appl Physiol 90:639-642

Hilberg T, Prasa D, Stürzebecher J, Gläser D, Schneider K, Gabriel HHW (2003b) Blood coagulation and fibrinolysis after extreme short-term exercise. Thromb Res 109:271-277

Hilberg T, Menzel K, Wehmeier UF (2013) Endurance training modifies exercise-induced activation of blood coagulation: RCT. Eur J Appl Physiol 113:1423-1430

Hood MS, Little JP, Tarnopolsky MA, Myslik F, Gibala MJ (2011) Low-volume interval training improves muscle oxidative capacity in sedentary adults. Med Sci Sports Exerc 43:1849-1856

Little JP, Gillen JB, Percival ME, Safdar A, Tarnopolsky MA, Punthakee Z, Jung ME, Gibala MJ (2011) Low-volume high-intensity interval training reduces hyperglycemia and increases muscle mitochondrial capacity in patients with type 2 diabetes. J Appl Physiol 111:1554-1560

Matsuda M, DeFronzo RA (1999) Insulin sensitivity indices obtained from oral glucose tolerance testing: comparison with the euglycemic insulin clamp. Diabetes Care 22:1462-1470

Matthews DR, Hosker JP, Rudenski AS, Naylor BA, Treacher DF, Turner RC (1985) Homeostasis model assessment: insulin resistance and $\beta$-cell function from fasting plasma glucose and insulin concentrations in man. Diabetologia 28:412-419

Menzel K, Hilberg T (2011) Blood coagulation and fibrinolysis in healthy, untrained subjects: effects of different exercise intensities controlled by individual anaerobic threshold. Eur J Appl Physiol 111:253-260

Nordestgaard BG, Benn M, Schnohr P, Tybjærg-Hansen A (2007) Nonfasting triglycerides and risk of myocardial infarction, ischemic heart disease, and death in men and women. JAMA 298:299-308

Posthuma JJ, van der Meijden PEJ, ten Cate H, Spronk HMH (2015) Short- and long-term exercise induced alterations in haemostasis: a review of the literature. Blood Rev 29:171-178

Reeves S, Halsey LG, McMeel Y, Huber JW (2013) Breakfast habits, beliefs and measures of health and wellbeing in a nationally representative UK sample. Appetite 60:51-57

Rognmo $\varnothing$, Moholdt T, Bakken H, Hole T, Mølstad P, Myhr NE, Grimsmo J, Wisløff U (2012) Cardiovascular risk of high- versus moderate-intensity aerobic exercise in coronary heart disease patients. Circulation 126:1436-1440

Silva KDRR, Kelly CNM, Jones AE, Smith RD, Wootton SA, Miller GJ, Williams CM (2003) Chylomicron particle size and number, factor VII activation and dietary monounsaturated fatty acids. Atherosclerosis 166:73-84

Siri WE (1956) The gross composition of the body. Adv Biol Med Phys 4:239-280

Tan MS, Mok A, Yap MC, Burns SF (2013) Effect of sprint interval versus continuous cycling on postprandial lipaemia. J Sports Sci 31:989-995 
Tan M, Chan Moy Fat R, Boutcher YN, Boutcher SH (2014) Effect of high-intensity intermittent exercise on postprandial plasma triacylglycerol in sedentary young women. Int J Sport Nutr Exerc Metab 24:110-118

Thompson PD, Franklin BA, Balady GJ, Blair SN, Corrado D, Estes NA, Fulton JE, Gordon NF, Haskell WL, Link MS, Maron BJ, Mittleman MA, Pelliccia A, Wenger NK, Willich SN, Costa F (2007) Exercise and acute cardiovascular events placing the risks into perspective: a scientific statement from the American Heart Association Council on Nutrition, Physical Activity, and Metabolism and the Council on Clinical Cardiology. Circulation 115:2358-2368

Trombold JR, Christmas KM, Machin DR, Kim IY, Coyle EF (2013) Acute high-intensity endurance exercise is more effective than moderate-intensity exercise for attenuation of postprandial triglyceride elevation. J Appl Physiol 114:792-800
Trost SG, Owen N, Bauman AE, Sallis JF, Brown W (2002) Correlates of adults' participation in physical activity: review and update. Med Sci Sports Exerc 34:1996-2001

Weiss C, Seitel G, Bärtsch P (1998) Coagulation and fibrinolysis after moderate and very heavy exercise in healthy male subjects. Med Sci Sports Exerc 30:246-251

Weiss C, Bierhaus A, Kinscherf R, Hack V, Luther T, Nawroth PP, Bärtsch P (2002) Tissue factor-dependent pathway is not involved in exercise-induced formation of thrombin and fibrin. $\mathrm{J}$ Appl Physiol 92:211-218

World Health Organisation (2011) Global atlas on cardiovascular disease prevention and control. World Health Organisation, Geneva, pp 8-11 (Available from World Health Organisation ) 